\title{
An investigation into the relation between step height and ground reaction forces in step exercise: a pilot study
}

\author{
Mark C Maybury, Jackie Waterfield
}

\begin{abstract}
The aim of this study was to investigate the effect that changing step height had on ground reaction force. Using a randomised crossover design, 12 volunteers with no previous experience of step aerobics were recruited to perform at three different step heights: 6,8 , and 10 inches. Subjects performed a basic step at a cadence of $120 \mathrm{beats} / \mathrm{min}$ and performed three one minute trials during which ground reaction force was measured. Measurement of peak impact force, time to achieve peak impact, and total time of foot contact was made, and impulse of the force was calculated. Statistically significant differences were found to exist for peak impact force between the 6 and 8 inch and 6 and 10 inch, but not between the 8 and 10 inch conditions. No significant differences were found in any other parameters. The study supports the present advice that participants should use low step heights, and possible mechanisms of injury are discussed. (Br F Sports Med 1997;31:109-113)
\end{abstract}

Keywords: step exercise; ground reaction forces; moments; eccentric contraction

This study investigates the relation between step heights and ground reaction forces. The hypothesis to be explored was that differences exist between three step height conditions-6, 8 , and 10 inches-in terms of peak vertical impact, impulse of the force, time to peak impact, and time of total foot contact. Literature relating to step exercise is reviewed, and the mechanisms of injury that are possibly associated with this type of activity are discussed.

Step aerobics is claimed to be a high intensity low impact aerobic workout, carrying a low injury risk, which conditions the lower body and can also condition the upper body. The intensity of the workout can be manipulated by adjusting step height and/or by the use of hand weights. Proponents of step aerobics claim that ground reaction forces (GRFs) are similar to those of walking. ${ }^{1}$ In a UK television consumers programme, ${ }^{2}$ step aerobics came under scrutiny because of these claims. Safety fears were expressed for participants because of claims that the research cited to authenticate step aerobics was inherently flawed. Injury potential arising from incorrect stepping techniques was also highlighted.

There are few reported studies on the effects of step aerobics on GRF. Johnson et al compared 40 minutes of bench stepping on an 8 inch step with "normal" walking, slow jogging, low impact marching, and high impact double hop knee lifts, and found that bench stepping had lower recorded GRFs (1.46 corrected for body weight (BW), 1.13BW, 2.26BW, 1.74BW and 3.14BW) than all other exercises, excluding walking. Farrington and Dyson $^{4}$ measured forces in three planes over four step heights: $4,6,8$, and 10 inches. They found that the vertical component of the GRF ranged from 1.453 to $1.863 \mathrm{BW}$ for the basic step; similar values were reported for other step patterns. They concluded that step aerobics may not be as low impact an exercise as originally thought, and they suggested that to minimise injury risk an optimal step height related to aerobic benefit could be used in combination with manipulation of the routine by the addition of hand weights and by using more complex step patterns.

Nisell et alp quantified ankle and knee moments, femoral shear, and compressive forces that occurred during four different step down activities from a $20 \mathrm{~cm}$ height. Their results indicated that step downs with the ball of the foot gave softer vertical GRFs, with lower forces recorded for backward ball steps than for forward ball steps. They went on to suggest that in forward jumps and steps the moment arms are almost equal for the vertical force $(\mathrm{Fz})$ and the anteroposterior force $(\mathrm{Fx})$ at the ankle joint. However, at the knee the Fx moment arm is relatively larger than the $\mathrm{Fz}$ moment arm, thus exerting a greater influence over knee load moment. Unfortunately they make no mention of forces related to backward steps.

Mital et at studied several riser and tread combinations to determine the values that gave minimal hip, knee, and ankle joint moments. They found that riser height had a profound effect on all moments, except hip moment. As the riser height increased, so did both knee and ankle joint force. Andriacchi $e t a l$ studied both ascending and descending stairs, and reported that descending brought about the largest moments, larger than walking. However, the mechanics of walking are different from those of ascending or descending stairs. In addition, phasic muscle activity is different, with increased activity in muscles responsible for vertical activity, and knee extensor force generated in stair climbing is higher than in level walking. 
Andriacchi et al suggest that if joint force is assumed to be proportional to external moment at the joint, then the magnitude of the knee joint force generated while descending stairs could be more than six times body weight at $50^{\circ}$ knee flexion. In walking, this amount of force tends to occur near full extension.

Nisell and Mizrahi ${ }^{8}$ studied the effects of different step down activities from step heights of 0.2 and $0.43 \mathrm{~m}$ ( 8 and 17 inches respectively). They reported that during step down activities the lowering leg is subject to more force than the impact leg, thus acting to decelerate and soften the impact of the lowered leg. When calculated, the compressive joint load when descending stairs was found to be four times body weight.

THE MUSCULOSKELETAL SYSTEM

A major role played by the musculoskeletal system is energy dissipation. During day to day activities joints and bones are subjected to high impulsive loads, leading to high peak dynamic loads. The idea that impulsive loads are involved in joint degeneration was proposed by Radin et al..$^{9}$ Joints are subjected to two types of force: shear force caused by articulation and compressive force produced by longitudinal loading. Further work by Radin and others ${ }^{10-12}$ suggests that degeneration of joints could be a consequence of repetitive loading, the nature and degree being more important than the total force. Gymnasts successfully attenuate landing forces by using large degrees of joint flexion, ${ }^{13}$ although the heights used in gymnastics are greater than those used in step aerobics. The body also relies on active and passive mechanisms. ${ }^{14}{ }^{15}$ Active attenuation, as in toe strike running, is achieved by proprioceptive activation of muscle tone to soften impact forces; this is also thought to lead to muscle and bone strengthening. ${ }^{16}$ Footwear also improves the shock attenuating ability of the body.$^{17}$ Pratt ${ }^{18}$ suggests that in modern running shoes forefoot cushioning can decrease by $20 \%$ after 350 miles of wear, even though rearfoot cushioning may be unaffected. He suggests that poor or worn shoes may give rise to patello femoral joint pain, peritendinitis of the tendo Achillis, and tibialis posterior tendinitis. In step aerobics, the ball of the foot is placed down first, followed by the heel. A loss of forefoot cushioning $^{14}$ would lead to reduced shock attenuation by the shoe material and increased shock attenuation by the body's active mechanisms, such as the intrinsic muscles of the foot and plantar flexors.

Stepping activities rely heavily on eccentric muscle work. Pain and stiffness are signs of muscle damage according to Strauber, ${ }^{19}$ and occur at different times after eccentric muscle action as a result of shortening of the non-contractile elements of the muscle. ${ }^{20}$ Muscle soreness can be classified into immediate and delayed, with maximum muscle trauma being caused by long duration high torque force producing exercises. ${ }^{21}$ This may be exacerbated by leg length inequality (anisomelia), which is often cited by some authors as a possible factor that may predispose individuals to an increased risk of injury. With injuries such as low back pain, stress fractures, and osteoarthritis, compensatory mechanisms and increasing internal joint forces can be introduced and these can be aggravated by repetitive impulse loading. ${ }^{22}$ Obviously, leg length inequality could have a bearing on injury incidence during step exercise, individuals with large discrepancies being more at risk.

\section{Methods}

To investigate the hypothesis, a randomised crossover design was implemented, using a Kistler force plate to measure GRFs. After satisfactory completion of a health screening questionnaire, a convenient self-selected sample of 12 female subjects were randomly assigned to three exercise sessions to perform at all the three heights under investigation. The three sessions were required to avoid warm up decrement and because of equipment and subject availability. The order in which the step heights were used by each subject was also randomised. Random assignment to the exercise sessions and to the order of step heights within each session was achieved by means of the random drawing of names. ${ }^{23}$ Novice steppers were used, experienced steppers being excluded from the study.

Variables thought to influence GRF were addressed in the following way.

- The stepping frequency was set at 120 beats/minute; lower stepping frequencies may have decreased, while higher frequencies may have increased, GRFs.

- No arm movements were taught, although they are often used in step classes to increase the work load, as these may have had an effect on GRF.

- Fatigue was prevented by the short duration of the exercise session; fatigue would have altered GRF and this factor was not under investigation because of limitations of time and equipment.

- Footwear type was limited to training shoes

- Practice effects were controlled by randomisation of the order of step heights for each subject.

A Reebok step was positioned adjacent to a Kistler force plate, type $9281 \mathrm{~B}$, sunk into a sprung walkway. This was to allow the subject to step one foot centrally on the force platform and the other on to the walkway (fig 1). The force plate was connected to a Kistler force plate control unit, and type 9851 data were collected by a BBC Master microcomputer with customised software. The ranges were set on the control unit at $2 \mathrm{kN}$ for vertical force $(\mathrm{Fz})$ and at $500 \mathrm{~N}$ for the horizontal force $(\mathrm{Fx})$ and anterior posterior force (Fy).

Before testing, heights and weights were measured (table 1), and subjects participated in a standardised warm up and stretching programme lasting 15 minutes. ${ }^{24}$ Only one subject had a leg length discrepancy of $1 \mathrm{~cm}$; this was measured from the inferior medial malleolus to the anterior superior iliac spine as advocated by McCaw and Bates. ${ }^{22}$

A two minute habituation period preceded the trials which consisted of three one minute 


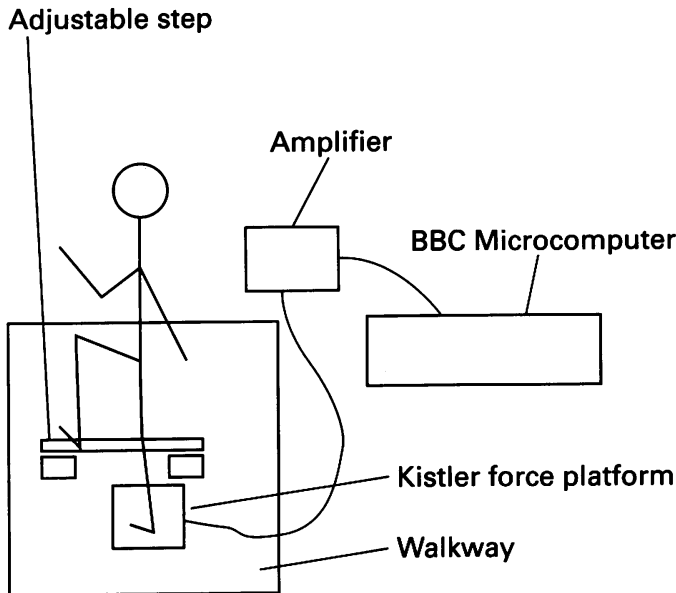

Figure 1 Diagrammatic representation of the position of the subject, step, and Kistler force plate.

Table 1 Subject characteristics

\begin{tabular}{llll}
\hline & Age (years) & Height $(\mathrm{cm})$ & Weight $(\mathrm{kg})$ \\
\hline Mean & 19 & 167.4 & 61.6 \\
Minimum & 17 & 158.5 & 50.6 \\
Maximum & 25 & 178.0 & 77.6 \\
\hline
\end{tabular}

stepping periods interspersed with 20 second intervals of marching on the top of the step. During the test, subjects stepped to a metronome rate of 120 beats/minute, corresponding to a stepping rate of $30 \mathrm{steps} /$ minute. On each initial step down, the Kistler force plate was triggered, the GRF was measured, and the forces were recorded. Data collection for the plate was set at one second time periods. It was decided beforehand that the second attempt would be taken for analysis; subjects were naive to this so as not to influence their stepping pattern in any way. The above procedure was adopted because of operating constraints of the force plate, thus enabling the force platform to be reset. The data collection sessions were video taped to assist in data interpretation; this also allowed stepping frequency to be monitored.

\section{STATISTICAL METHODS}

Data were analysed using a one way repeated measures analysis of variance, with $F$ corrected for heterogeneity of variance if necessary. The critical value for statistical significance was taken as $\mathbf{P}<0.05$. Impulse was calculated for each subject from a graph of applied force against time. Impulse reflects the momentum change that occurs during foot ground contacts.

\section{Results}

Table 2 gives a summary of the results. Analysis of the data for duration of foot contact at the various step heights showed no significant differences between the heights $(F=2.52$; df $=$ 22,$2 ; \mathrm{P}>0.05$ ).

Analysis of the data for time to achieve peak force at the various step heights showed no significant differences between the heights $(F=$ 1.98; $\mathrm{df}=22,2 ; \mathrm{P}>0.05$ ).

Analysis of the data for impulse at the various step heights showed no statistically significant differences between the heights $(F=$ $0.84 ; \mathrm{df}=22,2 ; \mathrm{P}>0.05$ ).

Analysis of the data for peak force at foot contact at the various step heights showed a significant within-subject effect $(F=8.67$; df $=$ $22,2 ; \mathrm{P}<0.01)$. Figure 2 shows a box and whisker plot of the peak force at the different step heights. Pairwise post hoc comparisons between the peak force scores at various step heights were carried out using Tukey's honestly significant difference (HSD) test for a repeated measures design. ${ }^{25}$ This procedure showed that the mean differences in peak force between 6 and 8 inches $(60.25 \mathrm{~N}, 95 \%$ confidence interval 17.28 to 103.22 ) and between 6 and 10 inches $(99.92 \mathrm{~N}, 95 \%$ confidence interval 40.25 to 159.58 ) were statistically significant, but that between 8 and 10 inches was not $(q=$ 2.935; $\mathrm{HSD}=50.147 ; \mathrm{P}<0.05)$.

\section{Discussion}

The results from this investigation appear to support the accepted practice that novices should use low step heights, as peak impact force increases with step height; this supports the work of Farrington and Dyson. ${ }^{4}$ However, there is still no conclusive evidence that GRFs are the main culprit in the pathomechanics of injury during step aerobics. Further investigations should be carried out to explore this.

The magnitude of force varied with step height. This accounted for the increase in impulse values, although a decrease in foot contact time was measured $(850,845$, and 820 $\mathrm{ms}$ respectively). It is interesting that the 8 inch step produced the greatest impulse at $426.2 \mathrm{~N}$, which could be explained by an observed hesitance by the subjects using the 10 inch step which did not appear in the other conditions. The time that the force acts in step exercise could easily have been attenuated by the body's active mechanisms in this latter condition.

It may be expected that impulse increases with body weight. This was found not to be the case, as the lightest subject did not produce the lowest peak impact force. Nisell and $\mathrm{Mizrahi}^{8}$ offer an explanation for this. During descension of stairs, the lowering leg softens the impact force of landing. Andriacchi et al report that knee moment forces on the lowering leg increase to up to six times body weight during

Table 2 Results for peak force, time to peak force, impulse, and foot contact

\begin{tabular}{|c|c|c|c|c|c|c|c|c|c|c|c|c|}
\hline & \multicolumn{3}{|c|}{ Peak force (N) } & \multicolumn{3}{|c|}{ Time to peak force (ms) } & \multicolumn{3}{|c|}{ Impulse $(\mathrm{N} / \mathrm{s})$} & \multicolumn{3}{|c|}{ Foot contact (ms) } \\
\hline & 6 inch & 8 inch & 10 inch & 6 inch & 8 inch & 10 inch & 6 inch & 8 inch & 10 inch & 6 inch & 8 inch & 10 inch \\
\hline Mean & 791.33 & 851.58 & 891.25 & 165.42 & 165.08 & 147.75 & 414.73 & 426.24 & 427.88 & 850.00 & 845.33 & 820.58 \\
\hline SD & 159.33 & 139.39 & 154.33 & 28.67 & 37.97 & 27.25 & 57.14 & 72.24 & 61.44 & 45.02 & 38.80 & 51.28 \\
\hline Minimum & 538.00 & 577.00 & 655.00 & 140.00 & 125.00 & 113.00 & 325.70 & 302.20 & 331.10 & 746.00 & 781.00 & 711.00 \\
\hline Maximum & 1038.00 & 1116.00 & 1163.00 & 234.00 & 260.00 & 218.00 & 513.70 & 519.70 & 506.80 & 933.00 & 910.00 & 890.00 \\
\hline
\end{tabular}




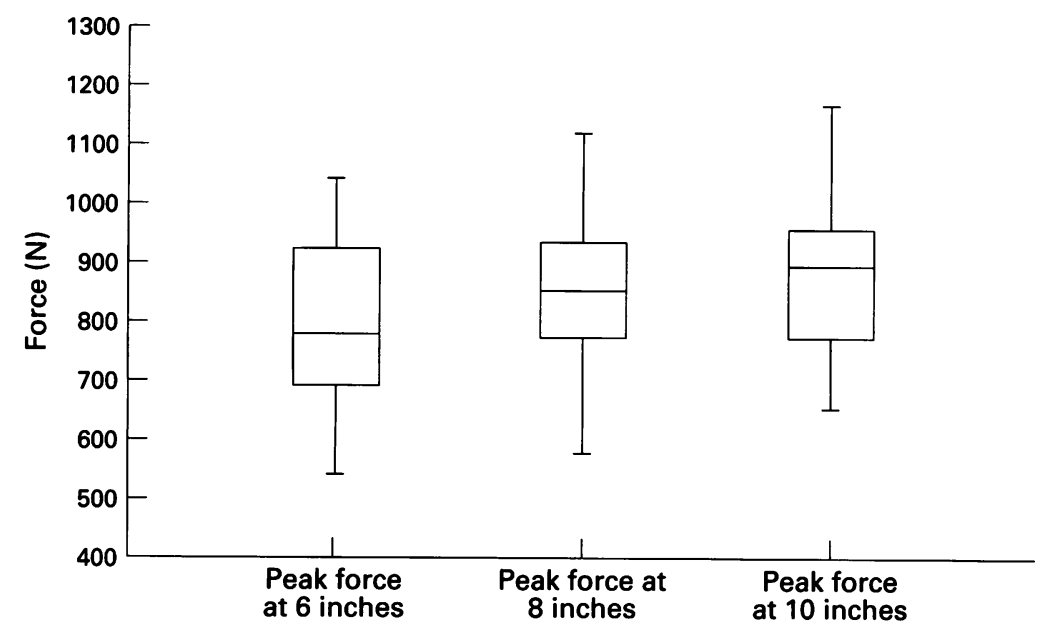

Figure 2 Box and whisker plot of peak force scores at 6, 8, and 10 inches. The horizontal band within the box denotes the median value, and the boundaries of the box represent the interquartile range. The whiskers indicate the total range of scores.

forward descension of stairs. However, in backward steps, moment forces are reduced compared with those calculated for forward stepping, because of the direction of the anteroposterior force; no magnitudes were given. Muscle activity also contributes to an increase in knee moment force. ${ }^{8}$ This, in addition to impulse loads, as suggested by Radin et $a l,{ }^{9}$ gives rise to a possible mechanism by which injury may occur during step. Mital et at suggested that during stair climbing, raising the step height increases the moment force about the knee joint. It is a reasonable assumption that, as the step height is increased, moment forces acting about the knee joint on both the lowering leg during step down and the leading leg during step up activities increase, leading to damage of the articular cartilage of the patellofemoral joint and tibiofemoral joint. Therefore impact forces may not necessarily be as important as moment forces in any possible mechanism of injury to both the lowering leg and the leading leg.

Parallels can be drawn between step aerobics and toe strike running, which places more stress on the plantar flexors of the ankle and flexors of the toes. However, toe strike running is only used for short periods of time to help cope with the increase in force that accompanies increased speeds; it could be theorised that the length of time spent stepping could cause inflammation of the flexor aponeurosis leading to plantar fasciitis. ${ }^{26}$ One might expect that throughout the whole of the step down sequence, shock absorption is under conscious control, the plantar flexors being preactivated to aid shock attenuation in the early stages, but as fatigue sets in the active attenuation may be overshadowed by passive mechanisms. Large joint flexions, particularly at the knee, may be used to assist in shock attenuation. ${ }^{13}$ It is this last point that is important when considering the effect of power steps, where the use of larger hip flexions would help to soften the impact of landing. Reebok's instructions to steppers ${ }^{1}$ advise participants not to step down with straight knees but to allow them to flex on landing.
FURTHER FACTORS THAT MAY BE RELATED TO INJURY IN STEP EXERCISE

Eccentric muscle activity is the predominant form of muscle contraction used in stepping. Essentially, a step class can be viewed as a prolonged activity that produces torque forces at the hip, knee, and ankle, ${ }^{68}$ which, according to Cleak and Eston, ${ }^{21}$ result in fatigue, maximal muscle soreness, and damage. Eccentric contractions produce greater muscular work than concentric contractions, but rely on the recruitment of fewer fibres. Evans ${ }^{27}$ supports this view and reports that fewer fibres are activated, resulting in muscle trauma caused by the generation of high intramuscular tensions. Eccentric muscle activity could potentially lead to damage of nervous tissue, especially at nervemuscle mechanical interfaces. Evidence that the main healing process within the muscle is through fibrosis is cited by Evans, ${ }^{27}$ and thus the nerve itself may become adhered to damaged muscle fibres giving rise to adverse mechanical tension type injuries.

Fatigue is a realistic problem that novice steppers have to cope with, which was controlled in this study by subjects stepping for five minutes only. However, it is common for novices to participate in a one hour class, of which 40 minutes would be stepping, consisting of both basic steps and more forceful steps. It is conceivable that, in the poorly conditioned, both cardiorespiratory fitness and local muscle endurance in the leg and thigh would be compromised leading to an increase in GRF and ultimately increasing the risk of injury. The concept of active and passive shock attenuation introduced by Nigg et al $^{14}$ gives an insight into the mechanism by which this could occur. Under the influence of fatigue the active mechanisms of shock attenuation could be reduced and the body would be more reliant on its passive mechanisms. Joints are prime areas to be damaged. Radin et al suggest that cartilage readily resists rubbing but is damaged by longitudinal compression. If the musculature became compromised by fatigue, the increased impact force may result in longitudinal compression leading to micro fracture of subchondral bone. This could be followed by destruction of the articular cartilage at the knee and possibly the hip, and eventually stress fractures could develop as the result of overuse. Injuries need not be confined to the lower limbs. According to Smeathers, ${ }^{28}$ force would be transmitted to the spine, and consequently degeneration of intervertebral joints and headaches may occur. In an individual with a latent mechanical back condition, this may cause untold damage from the increased shock attenuation that would be placed on the spine. Work by Ricard and Veatch ${ }^{16}$ indicates that passive impact forces can cause shearing and stretching of soft tissues, including nervous tissue.

Vertical force is believed to have the most influence on longitudinal loading. ${ }^{8}$ This may cause the horizontal and anterioposterior forces to be overlooked. These forces may become important in ankle injury as step height is increased. As the ankle moves from a 
loose packed position to a close packed position from toe down to heel down, it relies on surrounding muscular control for support. It has been observed that subjects hesitate before stepping down from a higher platform and this hesitation may result in a poorly braced ankle, leading to an ankle sprain. Fatigue could also exacerbate this.

Aging footwear may play a role in the mechanism of injury. The implication for step enthusiasts is that a reduction in forefoot cushioning may lead to an increased risk of injury, because it is the forefoot that contacts the ground first. In step exercise, the ball of the foot is placed down first, followed by the heel. A loss of forefoot cushioning ${ }^{14}$ would lead to reduced shock attenuation by the shoe material and increased shock attenuation by the body's active mechanisms, such as the intrinsic muscles of the foot and plantar flexors. This may lead to plantar fasciitis or peritendinitis of the tendo Achillis. Loss of rearfoot cushioning would not have such consequences, as explained by Lafortune and Henning. ${ }^{17}$

A factor that may influence the risk of injury is leg length. McCaw and $\mathrm{Bates}^{22}$ report that leg length inequality predisposes individuals to an increased risk of injury. It could be postulated that on stepping down, the shorter leg would travel slightly further before it contacted the ground, which would mean the longer lowering leg would be subjected to slightly higher moment forces which would cause increased damage to the patellofemoral joint. In addition, the resulting muscle imbalance in the pelvis, brought about by intrinsic compensations for the leg length inequality, may predispose the individual to lumbar spine problems.

It is recognised that this pilot study has limitations; the sample is not truly representative of the general population of step participants in terms of age, gender, and fitness levels. Further work may need to be carried out to investigate if stratifying for age and fitness level influences GRF in response to altering step height. Difficulties were observed in this study in step timing and the maintenance of correct technique which may need to be considered further, although this study did reflect what frequently happens with novice steppers. Errors may have also been introduced because of the orientation of the step platform.

\section{Conclusion}

The results of this study support the use of low step heights. Raising the step height appears to increase the forces exerted on the stepper, although no such relation was found to exist in terms of the impulse, time of foot contact, or time to achieve peak force. From the findings of this study, mechanisms of injury can be inferred. Eccentric muscle work predominates in step aerobics and this type of muscle contraction may lead to widespread muscle damage, producing adverse mechanical tension type injuries to nervous tissue. Fatigue, often experienced by novices, could lead to stress fractures in the tibia or calcaneus and an increased risk of osteoarthritis in later years. This is likely to arise through damage to articular surfaces caused by impulsive loads, related to the reduced effectiveness of the body's active shock attenuating mechanisms. However, patellofemoral and hip joint damage in stepping up and stepping down activities could be due to the moment forces about these joints in addition to GRFs. The relation between step height and moment forces warrants further investigation.

We would like to thank Dr Julius Sim, Principal Lecturer in Research Methods, Coventry University, for his statistical and general advice on this paper.

1 Reebok UK Ltd. Step Reebok Instructors Manual. London: Reebok, 1990

2 Robinson A. Watch Dog, BBC TV programme. Broadcast October 1993.

3 Johnson BF, Rupp JC, Berry SA, Rupp DA. Peak vertical ground reaction forces (PVGRFs) and time to peak force (TTPF) in bench stepping and other activities. Med $S c$ Sports Exerc 1992;24(suppl 131):783.

4 Farrington T, Dyson RJ. Ground reaction forces during step aerobics. Fournal of Human Movement Studies 1995;29:8998.

5 Nisell R, Mizrahi J, Ekholm J. Knee and ankle loads in step down activities. In: Jonsson $\mathrm{B}$, editor. International series on biomechanics. Biomechanics $X$. Champaign, IL: Human Kinetics Publishers, 1987:1137-42.

6 Mital A, Fard HF, Khaledi H. A biomechanical evaluation of staircase riser heights and tread depths during stair climbing. Clinical Biomechanics 1987;2:162-4.

7 Andriacchi TP, Andersson GBJ, Fermier RW, Stern D, Galente JO. A study of lower-limb mechanics during stairGalente JO. A study of lower-limb mechanics durin

8 Nisell R, Mizrahi J. Knee and ankle joint forces during steps and jumps down from two different heights. Clinical Bio mechanics 1988;3:92-100.

9 Radin EL, Parker HG, Pugh JW, Steinberg RS, Paul IL Rose RM. Response of joints to impact loading. III. Relationship between trabecular microfractures and cartilage degeneration. $\mathcal{F}$ Biomech 1973;6:51-7.

10 Anderson DD, Brown TD, Yang KH, Radin EL. A dynamic analysis of impulsive loading of the extension-splinted rabbit knee. F Biomech Eng 1990;112:119-28.

11 Farksa T, Boyd RD, Schaffler MB, Radin EL, Burr DB. Early vascular changes in rabbit subchondral bone after repetitive impulsive loading. Clin Orthop 1987;219:259-67.

12 Lukoschek M, Boyd RD, Schaffler MB, Burr DB, Radin EL Comparison of joint degeneration models. Surgical instability and repetitive impulsive loading. Acta Orthop Scand 1986;57:349-53.

13 McNitt-Gray J. Kinematics and impulse characteristics of drop landings from three heights. International fournal of Sport Biomechanics 1991;7:201-24.

14 Nigg, BM, Denoth J, Kerr S, Leuthi S, Smith S, Stacott A Load sports shoes and playing surfaces. In: Frederick EC editor. Sports shoes and playing surfaces. Champaign, IL Human Kinetics Publishers, 1984:1-23.

15 Jefferson RJ, Collins JJ, Whittle MW, Radin EL, O'Conno $\mathrm{JJ}$. The role of the quadriceps in controlling impulsive forces around heel strike. Proceedings of the Institution of mechanical Engineers. Part $H$. fournal of Engineering in Medicine 1990;204:21-8.

16 Ricard MD, Veatch S. Comparison of impact forces in high and low impact aerobic dance moves. International fournal of Sport Biomechanics 1990;6:67-77.

17 Lafortune MA, Henning EM. Cushioning properties of footwear during walking: accelerometer and force platform measurements. Clinical Biomechanics 1992;7:181-4

18 Pratt DJ. Mechanisms of shock attenuation via the lowe extremity during running. Clinical Biomechanics 1989;4:51 7

19 Strauber WT, Clarkson PM, Fritz VK, Evans WJ. Extracellular matrix disruption and pain after eccentric muscle action. $\mathcal{f}$ Appl Physiol 1990;69:868-74.

20 Newham DJ, Jones DA, Clarkson PM. Repeated high force eccentric exercise: effects on muscle pain and damage. Appl Physiol 1987;63:1381-6.

21 Cleak MJ, Eston RG. Delayed onset muscle soreness: Mechanisms and management. $\mathcal{f}$ Sports Sci 1992;10:32541.

22 McCaw ST, Bates BT. Biomechanical implications of mild leg length inequality. Br f Sports Med 1991;25:10-13.

23 Portney LG, Watkins MP. Foundations of clinical research. applications to practice. Norwalk: Appleton \& Lange, 1993.

24 Applications to practice. Norwalk: Appleton \& Lange, 1993. testing and prescription. 4th ed. Philadelphia: Lea \& Febiger, testing an

25 Hinton P. Statistics explained. London: Routledge, 1995.

26 Collins JJ, Whittle MW. Impulsive forces during walking and their clinical implications. Clinical Biomechanics 1989;4 179-87.

27 Evans WJ. Exercise induced skeletal muscle damage. Physician and Sports Medicine 1987;15:89-100.

28 Smeathers JE. Measurement of transmissibilty for the human spine during walking and running. Clinical Biomechanics 1989;4:34-40. 